\title{
Antibiotic Sensitivity of Strains of $E$. coli Isolated from Poultry Sheds Fed with Additive Saccharomyces cerevisiae and without Additive
}

\author{
Nora Guida*, Marcela Mascolo, Mariano Laiño, Carla Bustos, Pablo Franco \\ Catedra de Enfermedades Infecciosas, Facultad de Ciencias Veterinarias, Universidad de Buenos Aires, \\ Buenos Aires, Argentina \\ Email: ${ }^{*}$ guidanora@gamil.com
}

Received 14 February 2015; accepted 2 March 2015; published 6 March 2015

Copyright (C) 2015 by authors and OALib.

This work is licensed under the Creative Commons Attribution International License (CC BY). http://creativecommons.org/licenses/by/4.0/

(c) (i) Open Access

\begin{abstract}
The treatments against avian Colibacilosis have certain limitations, since the antimicrobial resistance has increased significantly and treatment may be ineffective. Prebiotics, administered as additives to animal, produce beneficial effects through changes in the microbial population of your digestive tract and which aims to ensure the normal balance of populations beneficial bacteria and dangerous to the digestive system. Yeasts such as Saccharomyces $(S$.) cerevisiae occupy sites of connection in the intestinal mucosa forming a physical barrier to potential pathogenic bacteria. Thus, these bacteria would be excluded by competition. The aim of this study was to assess the antibiotic sensitivity of strains of $E$. coli isolated from broiler sheds which used $S$. cerevisiae as an additive and others that did not use them. The $E$. coli strains were sensitive to cefotaxime, chloramphenicol and trimetoprimsulfa on samples for bird supplemented. In both groups there was a marked resistance to other antibiotics studied in this work.
\end{abstract}

\section{Keywords}

E. coli, Saccharomyces cerevisiae, Prebiotic, Poultry, Antibiotic

Subject Areas: Infectious Diseases, Veterinary Medicine

\section{Introduction}

Los antibióticos han sido ampliamente utilizados en la producción animal durante décadas. Aunque algunos se utilizan terapéuticamente para mejorar la salud y bienestar de los animales, la mayoría se utilizan con fines

"Corresponding author.

How to cite this paper: Guida, N., Mascolo, M., Laiño, M., Bustos, C. and Franco, P. (2015) Antibiotic Sensitivity of Strains of E. coli Isolated from Poultry Sheds Fed with Additive Saccharomyces cerevisiae and without Additive. Open Access Library Journal, 2: e1383. http://dx.doi.org/10.4236/oalib.1101383 
profilácticos, para mejorar la tasa de crecimiento y la eficiencia de conversión del alimento, como promotores del crecimiento (APC) [1].

Sin embargo, esta práctica está siendo cuestionada debido a la generación de genes de resistencia en las bacterias digestivas para los antibióticos utilizados en terapia humana y animal, una situación que podría representar un riesgo potencial para la salud pública.

Las enfermedades que afectan al sector avícola tienen gran influencia sobre los parámetros de producción o zootécnicos, y en algunos casos en salud pública. La falta de control sanitario o los tratamientos ineficaces en galpones de pollos de engorde conlleva a importantes pérdidas económicas por la mortalidad animal, retraso en el crecimiento, el costo del tratamiento veterinario, la disminución de la producción. Los gastos derivados del control de estas enfermedades suponen una gran inversión para la industria avícola.

Escherichia (E.) coli es la especie bacteriana predominante en la microbiota aerobia y anaerobia facultativas normal del tracto gastrointestinal de la mayoría de los animales y el hombre.

Las APEC (Avian Pathogen E. coli) pueden estar presentes en la microbiota normal del intestino y producir enfermedad colibacilar en los períodos en que las defensas de los animales se ven disminuidos.

La adherencia microbiana a los tejidos del huésped es un suceso crítico en la patogénesis de la mayoría de las infecciones. Las bacterias no adherentes se eliminan mediante los mecanismos de defensa no específicos del huésped como el peristaltismo, movimiento ciliar y el flujo de líquido. Los componentes microbianos que median la adhesión a los tejidos del huésped considerados como factores de virulencia son variados y complejos. Las adesinas o los pili manosa-sensibles o fimbrias tipo 1 son los factores mas extendidos entre las bacterias Gram negativas. Exhiben la propiedad de combinarse con los receptores celulares que contienen D-manosa que existen en un gran número de células, incluyendo fagocitos [2].

En las cepas APEC se detectaron varias moléculas de superficie, tales como fimbrias tipo 1. Un alto porcentaje de APEC tiene fimbrias tipo 1 y se sabe que su expresión está regulada por las condiciones del medio ambiente y los medios de cultivo [3] [4].

Los tratamientos contra la colibacilosis tienen ciertas limitaciones, ya que el número de cepas resistentes a los antimicrobianos ha aumentado mucho y los tratamientos puede ser ineficaces.

Es muy importante tener en cuenta que los brotes clínicos de la colibacilosis en aves de corral son causados por la acción combinada de varias cepas de E. coli.

Los prebióticos se han definido como una serie de sustancias o alimentos funcionales de una o varias especies microbianas u otros elementos orgánicos, que cuando se administran como aditivos en alimentos animales puede causar efectos beneficiosos sobre ellos por los cambios en la población microbiana en el tracto digestivo y tienen como objetivo garantizar el equilibrio normal entre las poblaciones de bacterias beneficiosas y perjudiciales en el tracto digestivo. Puede estar incluido en la preparación de una amplia gama de productos, incluyendo alimentos, medicinas y suplementos alimenticios [5].

El embrión es estéril hasta el nacimiento. Luego la colonización microbiana establecerá la microbiota intestinal antes de los siete días de vida.

Levaduras tales como S. cerevisiae, cuando se utilizan muertas, son capaces de atraer a las bacterias con fimbrias tipo I adheriendose las bacterias a los mananos de la pared celuar de la levadura y formar un conglomerado aglutinado que es "arrastrado" hacia la cloaca y expulsado con las heces. Forman asi una barrera física para las bacterias regulando la microbiota. Por lo tanto, estas bacterias serían excluidas por competencia. Este concepto se conoce con el nombre de "exclusión competitive”. La incorporación en la dieta de mananos-oligosacáridos derivados fosforilados de una cepa específica (Saccharomyces $(S)$ cerevisiae) tuvo el mismo efecto de exclusión competitiva [6]. Estas levaduras evitan la adhesión de los patógenos a las células epiteliales del intestino mediante el bloqueo de fimbrias tipo 1 que poseen los microorganismos, y que le permiten atacar la superficie del epitelio intestinal. Se encontró además que los mananos fosforilados tiene un efecto directo sobre las células inmunes del tracto gastrointestinal cuando son absorbidos por las células M dentro de las placas de Peyer, y mejora en las aves la integridad intestinal, ya que aumenta la altura de la vellosidad y da uniformidad [7]. Las paredes celulares de levadura de $S$. cerevisiae están siendo utilizados en las aves de corral durante más de una década, algunos de los propósitos de esta práctica son para mejorar la productividad y la salud del ave.

El objetivo de este estudio fue evaluar la susceptibilidad antimicrobiana de las cepas de E. coli aisladas de galpones de engorde que usaron $S$. cerevisiae como aditivo y galpones que no lo estaban usando. 


\section{Materiales y Métodos}

Se recibieron en el laboratorio muestras de intestinos obtenidos por necropsia de pollos de entre 15 y 25 días de edad y una muestra de la cama de cada galpón. Se estudiaron 3 muestras de 12 galpones cuyos animales recibían aditivo de $S$. cerevisiae en su dieta (Galpón de prueba) y 3 muestras de 4 galpones (Galpón control) que no recibían ningún aditivo y de granjas diferentes. El contenido de las asas intestinales se diluyeron en $5 \mathrm{ml}$ de agua estéril, se homogeneizó y todas las muestras se sembraron en placas de Petri estériles con Agar tripticasa de soja, Agar XLD y agar MacConkey (Britania ${ }^{\circledR}$ ) para aislamiento de E. coli. Se incubaron a $37^{\circ} \mathrm{C}$ durante 18 - 24 horas y tres colonias de cada cultivo compatibles con E. coli fueron aisladas. La identificación se realizo con los métodos de rutina según Cowan and Steel's [8]. Se obtuvieron en total 36 cepas de galpones de prueba y 12 cepas de galpones control.

Estudio de la sensibilidad a los antibióticos:

Se realizo un pool de $E$. coli aisladas de cada galpón, 12 mezclas de galpones de prueba y 4 mezclas de galpones control se realizo el antibiograma según Kirby-Bauer, Clínica and Laboratory Standards Institute (CLSI) [9] [10].

El ensayo consistió en probar 9 antibióticos factibles de ser utilizados en pollos como tratamiento medicamentoso: polimixina, tetraciclina, neomicina, cefotaxima, enrofloxacina, cloranfenicol, gentamicina, colistina Trimetroprimsulfa.

\section{Resultados}

Todos los aislamientos fueron resistentes a neomicina. Sobre la polimixina y tetraciclina solo un galpón de prueba evidenció sensibilidad y la colicistina, y gentamicina solo en un galpón control fueron sensibles.

La mayor sensibilidad fue para cefotaxima y cloranfenicol en los galpones de prueba, (tal vez por su uso no autorizado en animales cuya carne se destine a consumo alimentario humano) en los galpones de prueba. Trimetoprimsulfa demostró sensibilidad en ambos grupos (Table 1, Figure 1 y Figure 2).

Según los porcentajes obtenidos cloranfenicol y trimetoprimsulfa obtuvieron mayor sensibilidad con las cepas aisladas en galpones tratados (Table 2).

\section{Discusión y Conclusiones}

Se ha observado una alta resistencia a diferentes antibióticos tanto en el caso de los galpones de prueba como de control. Trimetoprimsulfa mostró mayor sensibilidad junto con cefotaxima en los galpones estudiados siendo

Table 1. Sensibilidad (s) y resistencia (R) de aislamientos de E. coli aisladas de galpones de pollos tratados con S. cerevisiae y grupo control.

\begin{tabular}{|c|c|c|c|c|c|c|c|c|c|c|c|c|c|c|c|c|}
\hline \multirow[t]{2}{*}{ Antibiotico } & \multicolumn{12}{|c|}{$\begin{array}{l}\text { Pool de } E \text {. coli aisladas de animales que consumieron S. cerevisiae } \\
\text { Grupo de prueba } \mathrm{n}=12\end{array}$} & \multicolumn{4}{|c|}{$\begin{array}{l}\text { Pool de } E \text {. coli aisladas } \\
\text { de animales que no } \\
\text { consumian } S \text {. cerevisiae } \\
\text { Grupo control } n=4\end{array}$} \\
\hline & 1 & 2 & 3 & 4 & 5 & 6 & 7 & 8 & 9 & 10 & 11 & 12 & 1 & 2 & 3 & 4 \\
\hline Polimixina & $\mathrm{s}$ & $\mathrm{R}$ & $\mathrm{R}$ & $\mathrm{R}$ & $\mathrm{R}$ & $\mathrm{R}$ & $\mathrm{R}$ & $\mathrm{R}$ & $\mathrm{R}$ & $\mathrm{R}$ & $\mathrm{R}$ & $\mathrm{R}$ & $\mathrm{R}$ & $\mathrm{R}$ & $\mathrm{R}$ & $\mathrm{R}$ \\
\hline Neomicina & $\mathrm{R}$ & $\mathrm{R}$ & $\mathrm{R}$ & $\mathrm{R}$ & $\mathrm{R}$ & $\mathrm{R}$ & $\mathrm{R}$ & $\mathrm{R}$ & $\mathrm{R}$ & $\mathrm{R}$ & $\mathrm{R}$ & $\mathrm{R}$ & $\mathrm{R}$ & $\mathrm{R}$ & $\mathrm{R}$ & $\mathrm{R}$ \\
\hline Gentamicina & $\mathrm{R}$ & $\mathrm{R}$ & $\mathrm{R}$ & $\mathrm{R}$ & $\mathrm{R}$ & $\mathrm{R}$ & $\mathrm{R}$ & $\mathrm{R}$ & $\mathrm{R}$ & $\mathrm{R}$ & $\mathrm{R}$ & $\mathrm{R}$ & $\mathrm{R}$ & $\mathrm{R}$ & $\mathrm{R}$ & $\mathrm{s}$ \\
\hline Tetraciclina & $\mathrm{s}$ & $\mathrm{R}$ & $\mathrm{R}$ & $\mathrm{R}$ & $\mathrm{R}$ & $\mathrm{R}$ & $\mathrm{R}$ & $\mathrm{R}$ & $\mathrm{R}$ & $\mathrm{R}$ & $\mathrm{R}$ & $\mathrm{R}$ & $\mathrm{R}$ & $\mathrm{R}$ & $\mathrm{R}$ & $\mathrm{R}$ \\
\hline Cefotaxima & $\mathrm{s}$ & $\mathrm{s}$ & $\mathrm{s}$ & s & $\mathrm{s}$ & $\mathrm{s}$ & $\mathrm{s}$ & $\mathrm{s}$ & $\mathrm{s}$ & $\mathrm{s}$ & s & $\mathrm{s}$ & $\mathrm{R}$ & $\mathrm{R}$ & s & $\mathrm{s}$ \\
\hline Enrofloxacina & $\mathrm{R}$ & $\mathrm{R}$ & $\mathrm{R}$ & $\mathrm{R}$ & $\mathrm{s}$ & $\mathrm{s}$ & $\mathrm{R}$ & $\mathrm{R}$ & $\mathrm{R}$ & $\mathrm{R}$ & $\mathrm{R}$ & $\mathrm{R}$ & $\mathrm{R}$ & $\mathrm{R}$ & $\mathrm{R}$ & $\mathrm{R}$ \\
\hline Cloranphenicol & $\mathrm{s}$ & $\mathrm{s}$ & $\mathrm{s}$ & $\mathrm{s}$ & $\mathrm{s}$ & $\mathrm{s}$ & $\mathrm{s}$ & $\mathrm{R}$ & $\mathrm{R}$ & $\mathrm{s}$ & s & $\mathrm{s}$ & $\mathrm{s}$ & $\mathrm{R}$ & $\mathrm{R}$ & $\mathrm{R}$ \\
\hline Trimetroprim sulfa & s & $\mathrm{s}$ & $\mathrm{s}$ & s & $\mathrm{s}$ & $\mathrm{s}$ & $\mathrm{R}$ & $\mathrm{s}$ & $\mathrm{s}$ & $\mathrm{s}$ & $\mathrm{s}$ & $\mathrm{s}$ & $\mathrm{s}$ & $\mathrm{s}$ & $\mathrm{s}$ & $\mathrm{R}$ \\
\hline Colisistina & $\mathrm{R}$ & $\mathrm{R}$ & $\mathrm{R}$ & $\mathrm{R}$ & $\mathrm{R}$ & $\mathrm{R}$ & $\mathrm{R}$ & $\mathrm{R}$ & $\mathrm{R}$ & $\mathrm{R}$ & $\mathrm{R}$ & $\mathrm{R}$ & $\mathrm{s}$ & $\mathrm{R}$ & $\mathrm{R}$ & $\mathrm{R}$ \\
\hline
\end{tabular}




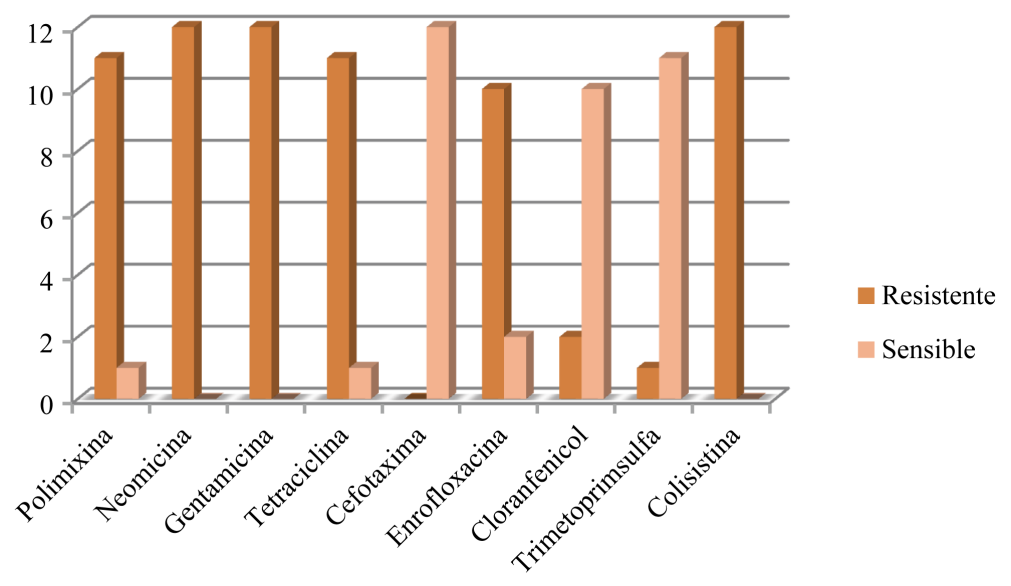

Figure 1. Sensibilidad y resistencia de aislamientos de E. coli aisladas de galpones de pollos tratados con S. cerevisiae.

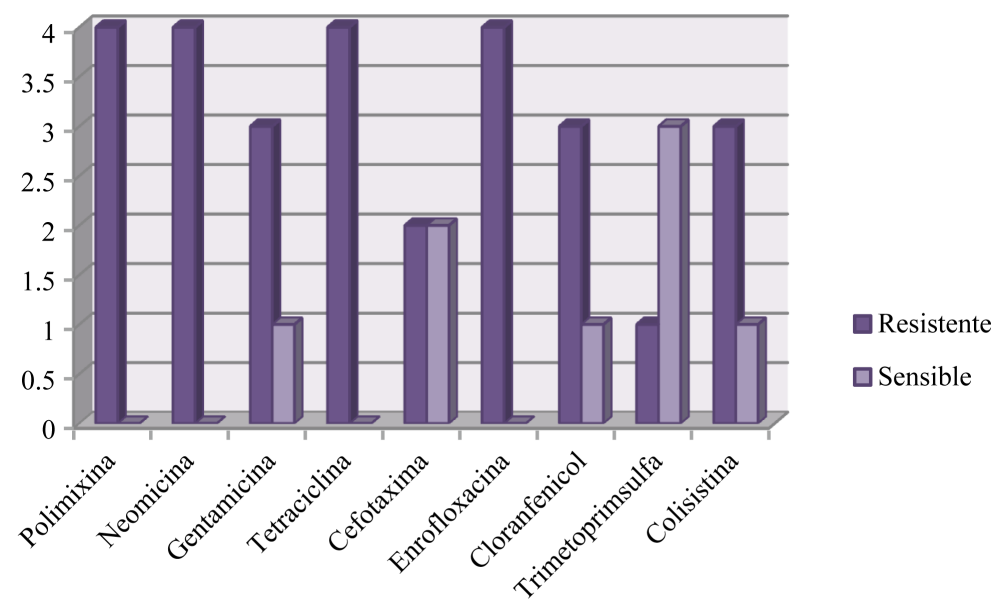

Figure 2. Sensibilidad y resistencia de aislamientos de E. coli aisladas de galpones de pollos del grupo control.

Table 2. Porcentaje de resistencia (R) a los antibióticos probados en ambos grupos.

\begin{tabular}{rcc}
\hline \multirow{2}{*}{ Antibiotico } & Grupo de prueba & Grupo control \\
\cline { 2 - 3 } Polimixina & Porcentaje de resistencia (\%) \\
Neomicina & 91.66 & 100 \\
Gentamicina & 100 & 100 \\
Tetraciclina & 100 & 75 \\
Cefotaxima & 91.66 & 100 \\
Enrofloxacina & 0 & 50 \\
Cloranphenicol & 83.33 & 100 \\
Trimetroprim sulfa & 16.66 & 75 \\
Colisistina & 8.33 & 25 \\
\hline
\end{tabular}


mayor el número de aislamientos sensibles en los galpones tratados. Otros autores realizaron un estudio para determinar la susceptibilidad bacteriana en aislamientos de humanos y animales encontraron una resistencia para E. coli del $31 \%$ y $49,1 \%$ para trimetoprimsulfa y $37 \%$ y $63,5 \%$ para cefalexina [11] [12].

Todas las muestras de $E$. coli aisladas en este trabajo fueron resistentes a más de 4 drogas antimicrobianas, es decir, que el $100 \%$ de ellas son multiresistentes, así podemos concluir que existen elevados porcentajes de resistencia de las cepas aisladas hacia los 9 antimicrobianos empleados en este trabajo.

El uso de los antibióticos en forma empírica puede generar una relación epidemiológica con el uso de antibióticos en la medicina humana y la cadena alimentaria humana y causar cepas multirresistentes a través de mutación genética [13]. Por otra parte la resistencia y multirresistencia de aislamientos de E coli de diversos estudios tanto en el hombre como animales es variable en los distintos países.

Para microbiólogo clínico es importante contar con estudios anteriores sobre la resistencia a los antibióticos contra diversas bacterias en diferentes especies animales para las pruebas de sensibilidad y asesorar al veterinario clínico sobre el uso inadecuado de antibióticos y optimizar su uso en caso de enfermedad.

Los antibióticos probados que resultaron ser más efectivos in vitro serían factibles de ser utilizados con mayor grado de certeza no observándose diferencia significativa en cuanto a la generación de resistencia en los galpones de prueba y los galpones control.

\section{References}

[1] Barrow, G.I. and Feltham, R.K.A., Eds. (1993) Cowan and Steel’s Manual for the Identification of Medical Bacteria. 3rd Edition, Cambridge University Press, Cambridge, 331 p. http://dx.doi.org/10.1017/CBO9780511527104

[2] Amabile De Campos, T., Guedes Stehling, E., Ferreira, A., Pestana de Castro, A.F., Brocchi, M. and Dias da Silveira, W. (2005) Adhesion Properties, Fimbrial Expression and PCR Detection of Adhesin-Related Genes of Avian Escherichia coli Strains. Veterinary Microbiology, 106, 275-285. http://dx.doi.org/10.1016/j.vetmic.2004.12.025

[3] Ozaki, H., Esaki, H., Takemoto, K., Ikeda, A., Nakatani, Y., Someya, A., Hirayama, N. and Murase, T. (2011) Antimicrobial Resistance in Fecal Escherichia coli Isolated from Commercial Chickens on Farms Growing Broiler. Veterinary Microbiology, 150, 132-139. http://dx.doi.org/10.1016/j.vetmic.2010.12.020

[4] Cinical and Laboratory Standards Institute (CLSI) (2008) Performance Standards for Antimicrobial Disk and Dilution Susceptibility Test for Bacteria Isolated from Animals. Approved Standard, M31 A3, Wayne, PA.

[5] Vidotto, M.C., Navarro, H.R. and Gaziri, L.C.J. (1997) Adherence Pili of Pathogenic Strains of Avian Escherichia coli. Veterinary Microbiology, 59, 79-87.

[6] Aguirre Alvarado, H., Plascencia Hernández, A., Rivera Mendoza, C.C., Guerrero Becerra, M. and Murillo Neri, V. (2007) Resistencia de Escherichia coli en infecciones de vías urinarias en pacientes pediátricos del Hospital Civil de Guadalajara "Fray Antonio Alcalde”. Enf Inf Microbiol, 27, 83-87.

[7] Brito, K.C.T., Jaenisch, F.R.F., Oliveira, G.A., Soares, B.D. and Brito, B.G. (2011) Resistencia antimicrobiana y patogenicidad de muestras de Escherichia coli aisladas de lesiones de celulitis en pollos XXII Congreso panamericano de avicultura, Bs As, Argentina. http://ainfo.cnptia.embrapa.br/digital/bitstream/item/54734/1/resistencia-antimicrobiana.pdf

[8] Sun, X., McElroy, A., Webb Jr., K.E., Sefton, A.E. and Novak, C. (2005) Broiler Performance and Intestinal Alterations When Fed Drug-Free Diets. Poultry Science, 84, 1294-1302. http://dx.doi.org/10.1093/ps/84.8.1294

[9] Spring, P., Wenk, C., Dawson, K.A. and Newman, K.E. (2000) The Effects of Dietary Mannanoligosaccharides on Cecal Parameters and the Concentrations of Enteric Bacteria in the Ceca of Salmonella-Challenges Broiler Chicks. Poultry Science, 79, 205-211. http://dx.doi.org/10.1093/ps/79.2.205

[10] Fedorak, R.N. and Madsen, K.L. (2004) Probiotics and Prebiotics in Gastrointestinal Disorders. Current Opinion in Gastroenterology, 20, 146-155. http://dx.doi.org/10.1097/00001574-200403000-00017

[11] Mosquito, S., Ruiz, J., Bauer, J.L. and Ochoa, T.J. (2011) Mecanismos moleculares de resistencia antibiótica en Escherichia coli asociadas a diarrea. Rev Peru Med Exp Salud Publica., 28, 648-656.

[12] Carranza, C. (2012) Caracterización y distribución de cepas de Escherichia coli potencialmente patógenas aisladas de pollos broiler de explotaciones avícolas en el Perú. Revista de Investigaciones Veterinarias del Perú, 23.

[13] Cinical and Laboratory Standards Institute (CLSI) (2011) Performance Standards for Antimicrobial Susceptibility Testing. M 100-S21, Wayne, PA. 